\title{
Emergency Messages Dissemination Challenges Through Connected Vehicles for Efficient Intelligent Transportation Systems: A Review
}

\author{
Muhammad Akram Mujahid ${ }^{1}$ \\ Fatima Tul Zuhra
}

\author{
Kamalrulnizam Abu Bakar ${ }^{1}$ \\ Muhammad Aamer Ejaz ${ }^{1}$
}

\author{
Tasneem S.J. Darwish ${ }^{2}$ \\ Gul Sahar
}

\footnotetext{
${ }^{1}$ School of Computing, Faculty of Engineering, Universiti Technologi Malaysia, Johor Bahru, Malaysia.

${ }^{2}$ Department of Systems and Computer Engineering, Carleton University, Canada.

*Corresponding author: akram1973@graduate.utm.my*, knizam@utm.my,tasneem83darwish@gmail.com, fatima-tulzuhra@utm.my, amerejaz555@gmail.com,gulsahar@kiu.edu.pk

"ORCID ID: https://orcid.org/ 0000-0002-0181-6664*,https://orcid.org/0000-0002-8771-9186, https://orcid.org/00000003-3942-2545, https://orcid.org/0000-0001-7767-1863, https://orcid.org/0000-0003-4069-3131, https://orcid.org/0000-0003-0225-4007
}

Received 8/10/2019, Accepted 1/6/2020, Published 1/12/2020

This work is licensed under a Creative Commons Attribution 4.0 International License.

\begin{abstract}
:
Recent growth in transport and wireless communication technologies has aided the evolution of Intelligent Transportation Systems (ITS). The ITS is based on different types of transportation modes like road, rail, ocean and aviation. Vehicular ad hoc network (VANET) is a technology that considers moving vehicles as nodes in a network to create a wireless communication network. VANET has emerged as a resourceful approach to enhance the road safety. Road safety has become a critical issue in recent years. Emergency incidents such as accidents, heavy traffic and road damages are the main causes of the inefficiency of the traffic flow. These occurrences do not only create the congestion on the road but also increase the fuel consumption and pollute the environment. Emergency messages notify the drivers about road accidents and congestions, and how to avoid the dangerous zones. This paper classifies the emergency messages schemes into three categories based on relay node, clustering and infrastructure. The capabilities and limitations of the emergency messages schemes are investigated in terms of dissemination process, message forward techniques, road awareness and performance metrics. Moreover, it highlights VANETbased challenges and open research problems to provide the solutions for a safer, more efficient and sustainable future ITS.
\end{abstract}

Key words: Accidents, Emergency messages, ITS, Road safety, VANET.

\section{Introduction:}

Intelligent Transportation System (ITS) is an integrated system of information and communication technologies used to improve the safety, mobility and efficiency of transportation network (1). ITS includes a wide range of applications that processes and shares information to improve the traffic management, ease congestion, minimize the environmental impact and increase the benefits of transportation users. The primary objective of the ITS is to improve the performance of the transport system by developing decision making systems to save lives, money, time, environment and energy (2). The conceptual model of the ITS is shown in the Fig 1.

ITS has several applications such as Arterial and Freeway Management (AFM), Freight Management (FM), Incident and Emergency Management
Systems (IEMS), Transit Management Systems (TMS), Information Management (IM) Systems, Emergency Vehicle Pre-emption (EVP), Highway Data Collection (HDC), Electronic Toll Collection (ETC), Traffic Management Systems (TMS), Vehicle Data Collection (VDC) and Transit Signal Priority (TSP) (3).

Traffic safety applications attract more attention because of more than 1,25 million deaths and 20-50 million critical injuries caused by road accidents worldwide each year (4). The main focus of the traffic safety applications is to send the messages with high reliability, low delay and maximize coverage by lowering dissemination cost. For safety messaging, the amount of information to be transmitted is relatively small, but the transmission 
reliability as well as the latency and packet dissemination are of great importance $(5,6)$.

Vehicular Network is the most significant and emerging technology in the field of ITS. VANET is considered as a baseline infrastructure for all applications and has attracted numerous researchers, scientists from both industry and the scholarly community everywhere throughout the world. In a VANET, moving vehicles as well as roadside infrastructure become nodes of a highly dynamic mobile network that can disseminate relevant information over long distances (7-9).

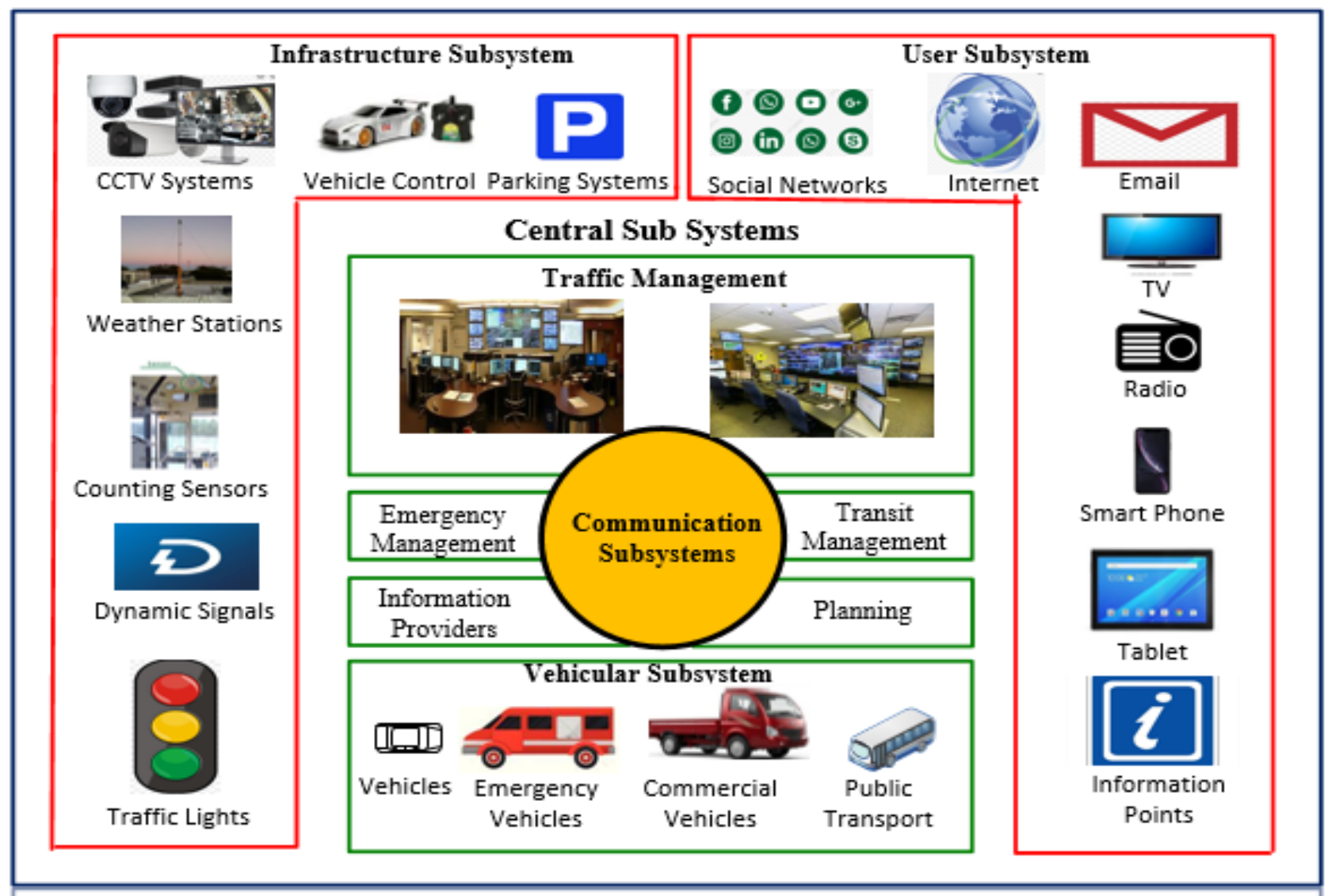

Figure 1. Conceptual Model of the ITS (10)

VANET is an innovation that uses moving vehicles as hubs to create a moving mobile network. Vehicles communicate with each other in the range of nearly 100 to 500 meters to exchange information. This type of communication depends on Vehicle to Vehicle (V2V) and Vehicle to Infrastructure (V2I) communication as shown in the Fig 2. IEEE $802.11 \mathrm{p}$ standard provides seven 10 $\mathrm{MHz}$ channels in the $5.9 \mathrm{GHz}$ licensed band for ITS applications with different channels designated for different applications (11-13).

The performance of the emergency messages schemes depends on the underlying VANET technology. The performance of these schemes is affected due to VANET characteristics such as frequent topological changes, high dynamicity, unexpected density variation, network disconnections and less connection time between vehicles (14).

Emergency incidents such as accidents, heavy traffic and road damages are the main causes of the inefficiency of the traffic flow. These occurrences do not only create the congestion on the road but also increase the fuel consumption and pollute the environment (15).

The majority of the EMs depends on broadcasting the messages which creates extra network overhead. Extra network overhead affects the performance of network by dropping packets and hence decreasing the packet delivery ratio. The effectiveness of the emergency messages schemes, in contrast with usual messages schemes, tightly bounds with reducing the delay while enhancing the reliability and increasing the packet delivery ratio $(16,17)$.

Several existing studies have tried to provide the real time traffic information about time sensitive incidents such as accidents by reducing the delay. Existing approaches are still facing a number of challenges in terms of maximum distance coverage, latency, reliability of the messages and emergency messages dissemination cost. 
Studying information dissemination techniques in VANET is an active research area where several review and survey papers have already been published on this topic. The data dissemination review (18) has categorized existing schemes based on opportunistic, vehicle-assisted and cooperative characteristics. Authors have discussed weaknesses and strengths of different broadcasting schemes. The focus of the survey is to highlight requirements for different types of application without focusing on inter-vehicles communications issues.

Hybrid information dissemination review (19) classifies dissemination schemes by considering push, pull and hybrid information distribution models. Communication overhead issues are highlighted while considering request-based and broadcast-based data disseminated approaches. The focus of this study is to highlight challenges based on scalability, security, trust, quality of service, node cooperation and simulation parameters.

The broadcasting based survey (20) divides the data dissemination schemes using broadcasting, multicasting and geocasting approaches. The author has discussed different disseminating techniques based on urban and highway scenarios. The focus of this study is to highlight bandwidth, communication overhead, maintenance, cost issues while maximizing information dissemination throughputs. The author focused on optimizing bandwidth requirements without prioritizing different ITS applications.

Hoping based review (21) has focused on classifying the broadcast dissemination schemes based on one hop, multihop, restrictive and promiscuous characteristics. The objective of this study is to analyze existing broadcast dissemination schemes based on same environment conditions, metrics and similar simulation platforms. Benefits and drawbacks of different schemes are studied based on different scenarios. The effects of dense and sparse network scenarios on the latency of warning messages are highlighted.

The work proposed by (22) has provided a complete taxonomy of information dissemination schemes based on security, quality of service, adaptive, timer, clustering, push, pull and hybrid features. The overall focus of this study is to highlight issues related to security and privacy, adaptability and scalability. The study has identified issues specific to requirement of each class. Open issues and challenges are also highlighted to improve the performance of information dissemination schemes.

Emergency message dissemination review (23) classifies the existing schemes based on Internet of things, priority messaging, clustering, software defined network, cellular networks and fog Computing. Proposed survey highlights the generic limitations of each category. Different schemes are analyzed based on delay, throughput, scalability, RSU, GPS and usage of technology. RSU Open issues are highlighted in the perspectives of normal city and smart scenarios.

These studies have classified the existing schemes based on techniques such as pull, push, opportunistic, multicasting, geocasting, QOS and a number of technologies including Internet of things, software defined network, cellular networks and fog Computing. Methodology proposed in each study has its pros and cons and improves performance under different scenarios and conditions. This paper classifies the existing emergency messages schemes into three main categories based on relay node, clustering and infrastructure. Moreover, it highlights the advantages and disadvantages of each type based on V2V and V2I communication.

This paper reviews the emergency messages schemes in context of the ITS to highlight the challenges that affects the performance of the ITS. The contributions of this review are as follows.

- The classification of the existing emergency messages schemes into three categories based on relay node, clustering and infrastructure.

- The capabilities and limitations of the emergency messages schemes are investigated in terms of dissemination process, message forward techniques, road awareness and performance metrics.

- Challenges and open research problems are highlighted that are needed to improve the performance of the ITS.

The rest of the paper is organized as follows: Section 2 classifies the existing work related to the distribution of the emergency messages. Section 3 summarizes the challenges and open research problems that should be considered for efficient ITS. Finally, the conclusion is presented in Section 4. 


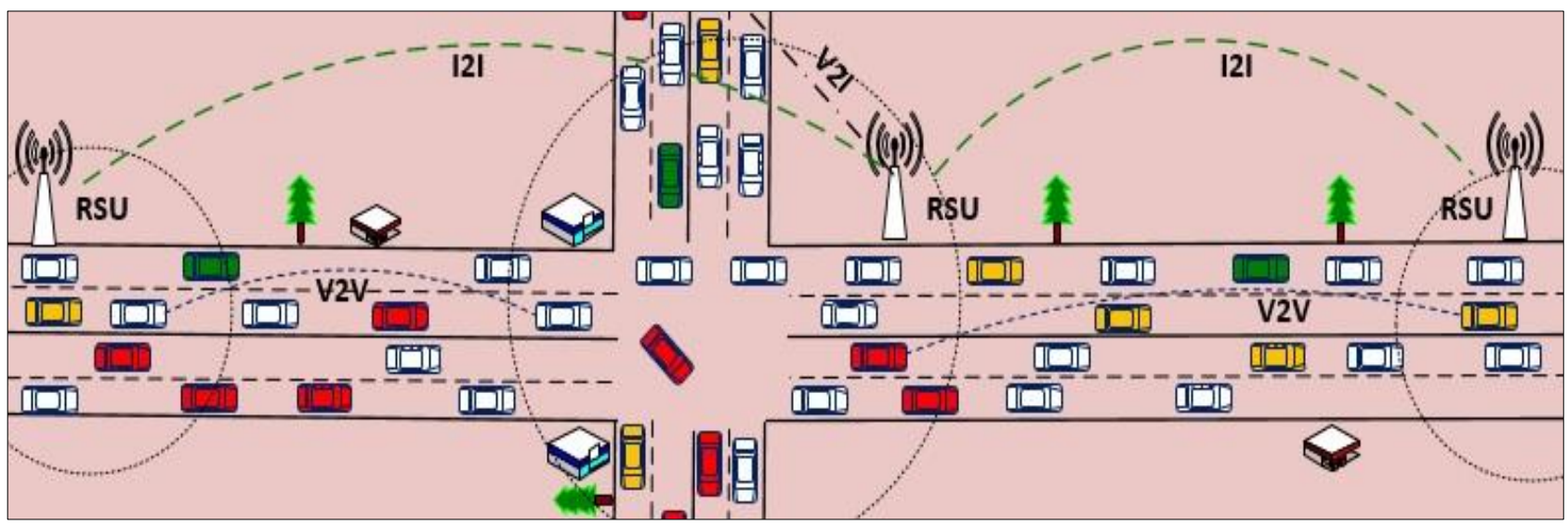

Figure 2. V2V and V2I Communications in VANET(24)

\section{Comparative Analysis of Existing Schemes:}

The emergency messages dissemination in VANET is the challenging task due to rapid and frequent topological changes. Broadcasting is the fundamental mechanism that is being used to disseminate the messages from one node to all other nodes. Broadcasting in VANET is specifically designed to support VANET safety applications. These safety applications require effective techniques to transmit data on the VANET. Emergency messages carry the information to update the network (25).

Emergency messages schemes consider broadcasting approach to disseminate the messages. Broadcasting depends on relay node, clustering and infrastructure such as RSU, ITL, ICU, sensors, central servers and cellular networks to disseminate the emergency messages. Based on these characteristics, existing emergency messages schemes are divided into relay node based, clustering based and infrastructure based as depicted in Fig 3. Although broadcasting mechanism is used in all categories but different techniques such as forwarder, clustering, RSU and cellular networks are used to increase the range of the emergency messages. Several recent studies regarding emergency messages are described in subsequent subsections.

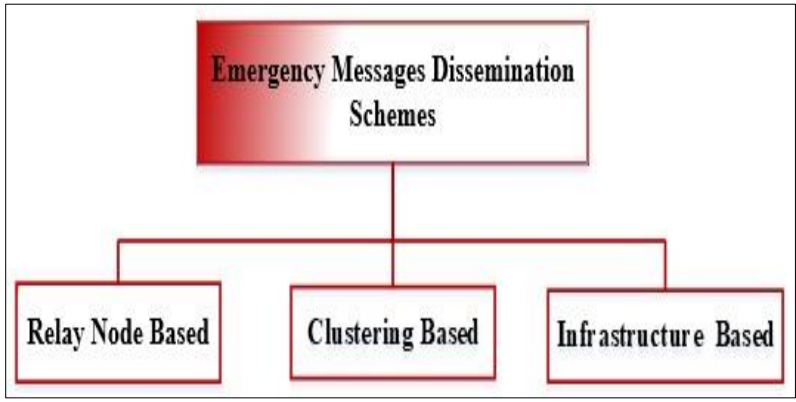

Figure 3. Classification of the existing emergency messages schemes.

\section{Relay Node-Based Schemes:}

In the relay node based schemes, a relay node is elected to broadcast the message. The election of relay nodes depends on a number of parameters such as density, distance and mobility $(26,27)$. The performance of the emergency messages schemes depends on the selection of the forwarder. The analysis of the relay node based schemes is given in the following section.

An Efficient Emergency Message Broadcasting (EEMB) scheme is proposed to avoid accidents and reduce congestion by selecting best relayers based on Link Life Time (LLT) (28).The focus of the scheme is to disseminate the warning message by reducing the overhead and increasing speed. Initially, affected vehicle choose the best relayer to broadcast the message. Subsequently, each relayer selects the forwarder to further propagate the message until the whole risk zone is covered as shown in Fig 4. Moreover, the proposed scheme tries to resolve the network fragmentation problem by using store-carry-forward technique. Although study tries to reduce the message delay by using $\mathrm{V} 2 \mathrm{~V}$ communication but in sparse network, the performance of the proposed solution will be affected. Along with that, the store-carry-forward approach creates extra delay in the propagation of the emergency messages. The election of a reliable relayer is also affected due to election from both directions.

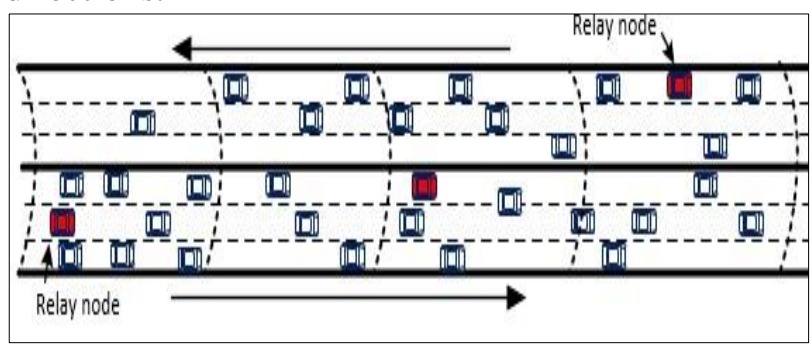

Figure 4. Dissemination of emergency messages using relay node (28) 
The best forwarder scheme (29) considers vehicle density to select the forwarder. To get density information, each vehicle uses beacon messages. The beacon messages include the vehicle ID and position. After receiving the beacon messages from neighbouring vehicles, each vehicle calculates the density and optimal distance from the current forwarder to itself. Then, a weighted formula is used to find optimum forwarder. Due to dependency on the higher density, extra network overhead is created which does not only increase the delay but also affects the range of the disseminated messages. While the reliable forwarder election scheme (30) considers proactive approach to elect the best forwarder, The Cooperative Neighbour Position Verification (CNPV) scheme depends on verifying the location of the neighbours before deciding the next forwarding vehicle. CNPV scheme does not only elect the forwarder but also detects nodes advertising false locations. Although reliability of the forwarder is improved but position verification before electing forwarder delays the election process which is not affordable in emergency messages schemes which depends on timely dissemination of the messages.

Moreover, the dynamic Partitioning Scheme (31) uses multihop message dissemination technique to forward the warning messages. A Forwarder node is elected whose distance from the source is maximum having large number of neighbour's nodes. The main focus of the scheme is to provide reliable, time-efficient, multihop dissemination while considering dense and light traffic scenarios. Due to varying speed of vehicles, dynamic partitioning creates overlapping areas, and network overhead is created which results in delaying the messages.

A distributed dissemination protocol (32) based on defining the Region of Interest (RoI) is also proposed. The selection of the next forwarder depends on distance and link quality. The main focus of this study is to reduce the risk of accidents while avoiding congestion in the affected area. Network load is also maintained to make efficient use of bandwidth while disseminating the messages in region of interests. Study reduces the network overhead by minimizing the ROI size which results in more number of segments. Consequently, the maximum distance coverage range is reduced and delay is increased.

A cross layer approach (26) depends on density, velocity and the geographical location of the vehicle to elect best forwarder. Data transmission rate is controlled using cross communication between layers. Proposed scheme elects a one-hop optimum forwarder for relaying the messages without defining broadcast range. It increases the number of forwarders which results in extra communication overhead.

As witnessed by the authors in (33), relay node is elected by using neighbour set ratio to reduce the route cost and delay. The total cost of each route is calculated using neighbour set ratio and mobility parameters. The main focus of this study is to reduce the drawbacks of the previous studies which utilize more transmission power and increase route discovery cost due to broadcast of hello messages. In high density area, the performance of the proposed scheme is affected due to high network overhead. Every vehicle having high neighbour set ratio advertises itself as optimum forwarder which compromises the reliability of the relay node.

A Prioritization and scheduled partitioning technique is proposed that depends on three phases to disseminate the messages (34). In the first phase, prioritization is performed. In the second phase, scheduled partitioning is used to broadcast the messages. Then the reliable transmission is completed in the last phase. Based on scenario of the Prioritized messages, sender and forwarder are elected. Adaptive scheduled partitioning creates overlapping areas which increases the communication overhead and limits the coverage range.

A local information sensing-based broadcast scheme (27) tries to address the issue of slow response and local broadcast storm. Source and forwarder embeds the topology information which includes effective candidate number, the effective communication distance, and traffic density before broadcasting the message. Receiver vehicles calculate the probability and set up a waiting time based on the information received from the sender. Characteristic information is affected due to rapid and frequent topological changes. Delay is incurred at sender and receiver side due to collection of characteristic information, setting up and waiting time.

The reliable emergency message dissemination scheme (35) has tried to optimize delay requirements while considering different conditions of the channels. Road awareness is also considered by dividing the road into segments and zones. Proposed scheme is based on the initialization phase and five key phases which include: data collection, local state processing, broadcast reliability guarantee, forwarders selection and cooperative one-hop reliability guarantee. The sender elects multiple forwarders on the basis of the best link reception quality. Although study elects multiple forwarders to avoid single point of failure, but this is done at the cost of a reliable forwarder. 
Overlapping areas will be crated which will reduce the range of the disseminated message. Kinetic theory based relay node selection (36) uses mobility prediction approach to improve the performance of the message dissemination. Kinetic degree of a node is estimated by predicting its future position and velocity. Best relay node has optimum value obtained through the combination of the three variables: coverage kinetic degree (CKD), kinetic distance to mean (KDTM) and signal power $\left(\mathrm{p}_{\mathrm{w}}\right)$. The major focus of the proposed scheme is to reduce the redundancy and delay while improving the reliability of the disseminated messages. As the proposed scheme is based on prediction, the stability and size of the relay segment are minimized due to sudden and unpredictable behaviour of vehicles. Network overhead also increases which affects the performance of the message dissemination scheme.

In the relay node based emergency messages dissemination schemes, forwarder is elected on the basis of distance, speed, location, neighbouring vehicles, neighbour set ratio and link life time. The main objective of the schemes is to provide the reliable dissemination to cover maximum vehicles with minimum delay. Existing studies enhance the performance of the emergency messages schemes but still there are some limitations. These limitations include runtime identification of the segment of the road in which relay node lies. There is still some work required to improve the existing studies to decide who will elect the next reliable forwarder: a sender vehicle or a receiver vehicle. Similarly, it requires the optimization of the election parameters to elect the most efficient forwarder. Schemes are needed to enhance the reliability of forwarders. In dense environment due to high density in a certain area, the number of vehicles increases. Election of forwarder is a challenging issue in this scenario. Likewise, in sparse network due to non-availability of forwarder, the delay increases. There is need to improve the existing schemes in different scenarios such as dense and sparse environment, and in different road sections such as intersections, blind curves, highways and urban roads.

The analysis of the relay node based schemes is given in Table 1.

Table 1. Comparative Analysis of Relay Node Based Emergency Messages Schemes

\begin{tabular}{|c|c|c|c|c|c|c|}
\hline $\begin{array}{c}\text { Year/ } \\
\text { Reference }\end{array}$ & Parameters & Scenario & $\begin{array}{c}\text { Road } \\
\text { Awareness } \\
\end{array}$ & Simulators & Performance Metrics & Weakness \\
\hline $2013[28]$ & Link life time & Highway & No & MATLAB & Latency, Overhead & $\begin{array}{l}\text { High delay, } \\
\text { reliability }\end{array}$ \\
\hline $2014[29]$ & Density & Highway & NO & NS2 & $\begin{array}{c}\text { Broadcast delay, Broadcast } \\
\text { count, }\end{array}$ & $\begin{array}{l}\text { Overhead, limited } \\
\text { distance coverage }\end{array}$ \\
\hline $2014[30]$ & Position & Urban & No & $\begin{array}{l}\text { NS2, CityMo } \\
\text { b }\end{array}$ & $\begin{array}{c}\text { Overhead, Warning } \\
\text { notification time, }\end{array}$ & High delay \\
\hline $2015[31]$ & $\begin{array}{l}\text { Location, } \\
\text { density }\end{array}$ & Highway & Yes & $\begin{array}{l}\text { OMNET++, } \\
\text { Sumo,veins }\end{array}$ & $\begin{array}{l}\text { Delay, Message delivery ratio, } \\
\text { Message dissemination speed }\end{array}$ & Overhead, delay \\
\hline $2015[32]$ & $\begin{array}{l}\text { Distance, link } \\
\text { quality }\end{array}$ & Urban & Yes & NS3, SUMO & $\begin{array}{l}\text { Packet delivery ratio, end-to- } \\
\text { end delay, overhead }\end{array}$ & $\begin{array}{c}\text { Delay, Limited } \\
\text { message } \\
\text { dissemination range }\end{array}$ \\
\hline $2017[26]$ & $\begin{array}{l}\text { Density, } \\
\text { velocity, } \\
\text { location }\end{array}$ & Urban & Yes & NS2 & $\begin{array}{c}\text { Delay, packet delivery ratio, } \\
\text { broadcast count }\end{array}$ & $\begin{array}{l}\text { Overhead, limited } \\
\text { message } \\
\text { dissemination range }\end{array}$ \\
\hline $2018[33]$ & $\begin{array}{l}\text { Neighbor set } \\
\text { ratio }\end{array}$ & Urban & NO & NS2 & $\begin{array}{l}\text { Packet delivery ratio, End to } \\
\text { end delay and route cost. }\end{array}$ & Delay, reliability \\
\hline $2018[34]$ & Position & Urban & Yes & Java & $\begin{array}{l}\text { Success ratio, Dissemination } \\
\text { efficiency, delay }\end{array}$ & $\begin{array}{c}\text { Overhead, coverage } \\
\text { range }\end{array}$ \\
\hline $2019[27]$ & $\begin{array}{l}\text { Distance, } \\
\text { density, } \\
\text { distribution } \\
\text { of neighbour }\end{array}$ & Urban & Yes & NS2, SUMO & $\begin{array}{l}\text { Packet Delivery Ratio } \\
\text { End-to-End Delay } \\
\text { Broadcast Redundancy } \\
\text { Forwarding Efficiency }\end{array}$ & Delay \\
\hline $2019[35]$ & $\begin{array}{l}\text { Link } \\
\text { reception } \\
\text { quality, } \\
\text { position }\end{array}$ & Highway & No & $\begin{array}{l}\text { OMNET++, } \\
\text { Veins }\end{array}$ & $\begin{array}{l}\text { Reliability, Network load, } \\
\text { delay }\end{array}$ & $\begin{array}{l}\text { Reliability, } \\
\text { coverage range }\end{array}$ \\
\hline $2020[36]$ & $\begin{array}{c}\text { Kinetic } \\
\text { distance, } \\
\text { degree, power }\end{array}$ & Urban & No & Matlab & $\begin{array}{c}\text { Forward size, duplicate } \\
\text { packets, delay, PDR and } \\
\text { overhead }\end{array}$ & Delay \\
\hline
\end{tabular}




\section{Cluster-Based Schemes:}

Cluster-based data dissemination depends on Cluster Head $(\mathrm{CH})$ to disseminate the message to its Cluster Members (CMs). $\mathrm{CH}$ use one to many approach to communicate with its CMs. Cluster head is elected using different parameters such as distance, speed, direction, link life time and density $(37,38)$. The working mechanism of clustering approach is given in Fig 5.

Cluster-based broadcast storm control scheme (37) is based on two phases: setup phase and steady-state phase. In the setup phase, two $\mathrm{CHs}$ are elected, one for each forward and backward direction based on the higher and lower speed of the vehicles. In the steady-state phase, further $\mathrm{CHs}$ are elected using speed, distance and number of warnings messages. $\mathrm{CHs}$ relay the message until new $\mathrm{CHs}$ are elected. In the proposed scheme, it is tried to reduce the broadcast storm by increasing $\mathrm{CHs}$. $\mathrm{CHs}$ are elected in both phases, due to which communication overhead is created which affects the performance of the message dissemination.

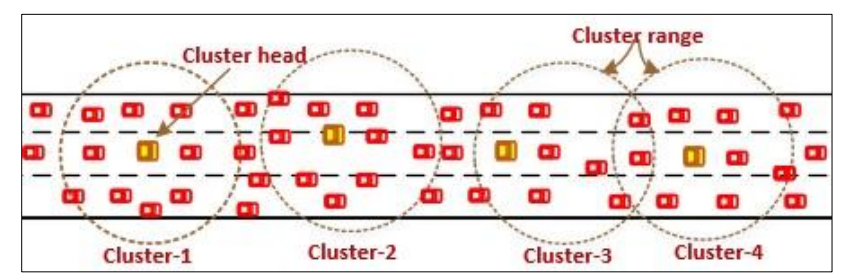

Figure 5. Clustering approach to disseminate the emergency messages (39)

Emergency message dissemination and collision avoidance scheme (40) uses a $\mathrm{CH}$ for the intra cluster communication between $\mathrm{CMs}$. A CH allocates time slots for its CMs. Moreover, a $\mathrm{CH}$ elects one of its $\mathrm{CM}$, whose position from the $\mathrm{CH}$ is optimum, as a Cluster Forwarder (CF). A CF is used to forward safety messages using backward control channel (CRL). Reliability of the proposed scheme is achieved through the use of two Media Access Control (41) layer protocols, Request-toBroadcast (RTB) and Clear to Broadcasts (CTB). A $\mathrm{CH}$ changes frequently which also affects the $\mathrm{CF}$ election. Extra communication overhead is created during $\mathrm{CH}$ and $\mathrm{CF}$ election which delays the process of emergency messages dissemination.

The scheme proposed by (42) uses dynamic power transmission and contention window size to avoid collisions during critical message dissemination. $\mathrm{CH}$ and sub $\mathrm{CH}$ election is based on number of vehicles without considering mobility parameters. $\mathrm{CH}$ notifies its cluster members about abnormal activity of the vehicles. Each vehicle dynamically adjusts broadcast power and contention window size to accommodate different types of messages. In the proposed scheme, it is tried to reduce the collisions. $\mathrm{CH}$ and sub $\mathrm{CH}$ election process does not only increase the communication overhead but also compromises the reliability of the disseminated messages.

Cluster and time barrier-based scheme (43) depends on the election of super vehicle to disseminate the emergency messages. A farthest vehicle from the source vehicle is designated as Super vehicle which will rebroadcast the emergency message after calculating the time barrier. Although researcher of the current work has tried to reduce the delay by increasing the coverage distance but it requires distance calculation of each vehicle with the source vehicle and distance comparison to elect super vehicle. This incurs extra communication overhead. Similarly, it is based on the election of farthest vehicle from the $\mathrm{CH}$ due to which the packet loss ratio is increased.

A clustering and position based scheme is proposed (44) to overcome the broadcast storm while reducing the communication delay. $\mathrm{CH}$ election is based on the probability of successful packet transmissions, channel fading and interest compatibility. $\mathrm{CH}$ sends the emergency messages to the vehicle moving into opposite direction of the current cluster. Every cluster member, after getting the emergency messages, relays to the $\mathrm{CH}$. The $\mathrm{CH}$ elects a cluster member to forward the message. A cluster member cannot forward the message on its own behalf. Because message is first forwarded to the $\mathrm{CH}$, the $\mathrm{CH}$ elects a cluster member to send the received message which incurs delay. Moreover, probability based schemes create extra delay and extra communication overhead is created during the selection of $\mathrm{CH}$ and message forwarding vehicles.

A clustering and warning messages prioritization scheme (4) considers inter-cluster and intra-cluster clock synchronization approach to make efficient use of channel utilization. Messages are prioritized based on three levels: by considering direction, message type and severity level. The $\mathrm{CH}$ election is based on the distance. The medoid node from a group of vehicles is elected as a $\mathrm{CH}$. Proposed scheme improves the channel utilization and reduces the communication by using clustering approach. The performance of the proposed schemes depends on the stability of the cluster. A $\mathrm{CH}$ is elected without considering mobility parameters. One of such parameters is speed. The $\mathrm{CH}$ will change frequently which creates extra communication overhead.

An adaptive fuzzy-based message dissemination scheme (45) uses fuzzy logic and micro artificial bee colony algorithm to broadcast the emergency messages. Proposed scheme is based on cluster 
formation, $\mathrm{CH}$ election, emergency message dissemination and route selection phases. Fuzzybased Decision Making Trial and Evaluation Laboratory (DEMATEL) approach is used to elect $\mathrm{CH}$. Micro Artificial Bee Colony algorithm (MABC) is used to define optimum routes. In the proposed scheme, it is tried to optimize the delay, throughput and packet delivery ratio. The $\mathrm{CH}$ election and optimum route election before sending the emergency messages delay the dissemination process.

Clustering approaches make use of $\mathrm{CH}$ to distribute the emergency messages to its cluster members. Scalability and channel access management are improved through the use of clustering approach. One of the main requirement of cluster-based approach is the formation of the clusters after the emergency situation occurred. Vehicles exchange messages to elect a reliable $\mathrm{CH}$ using election mechanisms. There is need of a chain of clusters to disseminate the information through clustering to cover the large area of the road. It does not only create extra communication overhead but also affects the other applications running on the network and incurs delay. Similarly, due to high dynamic nature of VANET networks, cluster instability occurs due to frequent change of $\mathrm{CH}$, non-optimum $\mathrm{CH}$ election parameters, frequent joining and leaving of cluster members, cluster member affiliation parameters, cluster breakage in sparse network and clustering overloading in dense environment. Additionally, existing studies are needed to be improved to reduce the inter cluster communication delay. Selection of reliable $\mathrm{CH}$ to disseminate the accurate information is also another challenge. The analysis of the clustering-based schemes is given in Table 2.

Table 2. Comparative Analysis of Cluster-based Emergency Messages Schemes

\begin{tabular}{|c|c|c|c|c|c|}
\hline $\begin{array}{c}\text { Year/ } \\
\text { Reference }\end{array}$ & Scenario & $\begin{array}{c}\text { Road } \\
\text { Awareness }\end{array}$ & Simulators & Performance Metrics & Weakness \\
\hline 2013 [37] & Highway & No & NS3 & $\begin{array}{l}\text { No of collisions, Success } \\
\text { rate and latency }\end{array}$ & $\begin{array}{l}\text { Communication } \\
\text { overhead, delay }\end{array}$ \\
\hline $2017[40]$ & Urban & Yes & NS2 & $\begin{array}{c}\text { Packet delivery ratio, delay, } \\
\text { throughput }\end{array}$ & $\begin{array}{c}\text { Frequent } \mathrm{CH} \text { change, } \\
\text { Delay }\end{array}$ \\
\hline $2017[41]$ & Urban/Highway & Yes & $\begin{array}{l}\mathrm{C}++, \mathrm{LTE} \\
\text { Framework }\end{array}$ & Delay, Resource usage & Costly \\
\hline $2017[42]$ & Highway & NO & NS2 & $\begin{array}{l}\text { Delay, Delivery Ratio, } \\
\text { Energy, Throughput }\end{array}$ & $\begin{array}{c}\text { Communication } \\
\text { overhead, reliability }\end{array}$ \\
\hline $2019[43]$ & Urban & No & $\begin{array}{l}\text { OMNET++, } \\
\text { SUMO }\end{array}$ & $\begin{array}{l}\text { Coverage area, Delay, } \\
\text { Successful transmission, } \\
\text { Impact of beacon interval } \\
\text { on EM. }\end{array}$ & $\begin{array}{l}\text { High packet loss } \\
\text { ratio, delay }\end{array}$ \\
\hline $2019[44]$ & Urban & No & $\begin{array}{l}\text { OMNET++, } \\
\text { SUMO }\end{array}$ & $\begin{array}{l}\text { Information coverage, } \\
\text { Transmission delay, PDR }\end{array}$ & $\begin{array}{l}\text { Communication } \\
\text { overhead, delay }\end{array}$ \\
\hline $2020[4]$ & Highway & No & $\begin{array}{l}\text { VANET } \\
\text { Toolbox }\end{array}$ & $\begin{array}{c}\text { Delay, Message loss rate, } \\
\text { Throughput }\end{array}$ & $\begin{array}{c}\text { Frequent } \mathrm{CH} \text { change, } \\
\text { Communication } \\
\text { overhead }\end{array}$ \\
\hline $2020[45]$ & Highway & No & MATLAB & $\begin{array}{c}\text { Throughput, packet } \\
\text { delivery ratio, delay, } \\
\text { overhead }\end{array}$ & Delay \\
\hline
\end{tabular}

\section{Infrastructure-Based Schemes:}

In infrastructure-based data dissemination, message is broadcasted to all nodes in its range using some sort of infrastructure such as RSU, ITL, ICU, sensors, central servers and cellular networks (46-48). Some infrastructure-based schemes are focused on providing the complete solution in terms of accident prevention, prediction of accident, congestion management and rerouting of traffic after accident. One of the key functions in these systems is to disseminate the emergency messages. The infrastructure approach is given in Fig 6 .
The RSU based scheme (49) is based on three types of the information such as weather information obtained through sensors, vehicles density information obtained through vehicles and accidents information through sensors installed on vehicles. Vehicles update the current status to the nearest RSU. The RSU processes this information and compares it with normal values. In case of abnormal values, RSU broadcasts the information to vehicles in its proximity and to other RSUs. In this scheme, two types of messaging are involved: from vehicles to RSU and from RSU to vehicles. The messages initiated from the RSU after processing 
have higher priority. Usage of the bandwidth is reduced by prioritizing the messages. This scheme utilizes the RSU to process and broadcast the emergency messages. To get the maximum benefits, roads should be populated with RSU, which will be a very costly solution. Along with this cost issue, the broadcast of the emergency message from source RSU and neighbouring RSU will populate the network with emergency messages which ultimately will slow down the message delivery process.

A Collision Avoidance scheme (50) uses RSU to divide the affected area into small segments. The source vehicle which had an accident sends the message to its nearest segment. This scheme tries to reduce the RSU usage by exploiting V2V communication. Although author has tried to improve the performance by using minimum resources but in sparse network area, the performance of the proposed algorithm will be affected. Additionally, a mechanism is required to divide the area into small segments. It is not clearly defined whether these segments will be static or dynamic. Likewise, a criterion will be required to divide the road into segments. This division will also slow down the process of message delivery.

The warning message dissemination scheme (46) uses Intelligent Traffic Lights (ITL). ITL are deployed at every intersection. These ITL update drivers about the current status of the traffic and also guides the drivers to use alternate paths. Every vehicle updates its neighbour's information to ITL. ITL calculate the density of the traffic based on the received information. Due to the overlapping communication range of vehicles, there is a chance that some vehicles may be counted more than once, which will give wrong information about the density of vehicles. Proposed algorithm seems to be feasible on highway environment, but in the case of urban area, it is very challenging to deploy ITLs at every intersection. Similarly, every vehicle is updating traffic information to the nearest ITL which creates communication overhead and affects the performance of the delivery of messages.

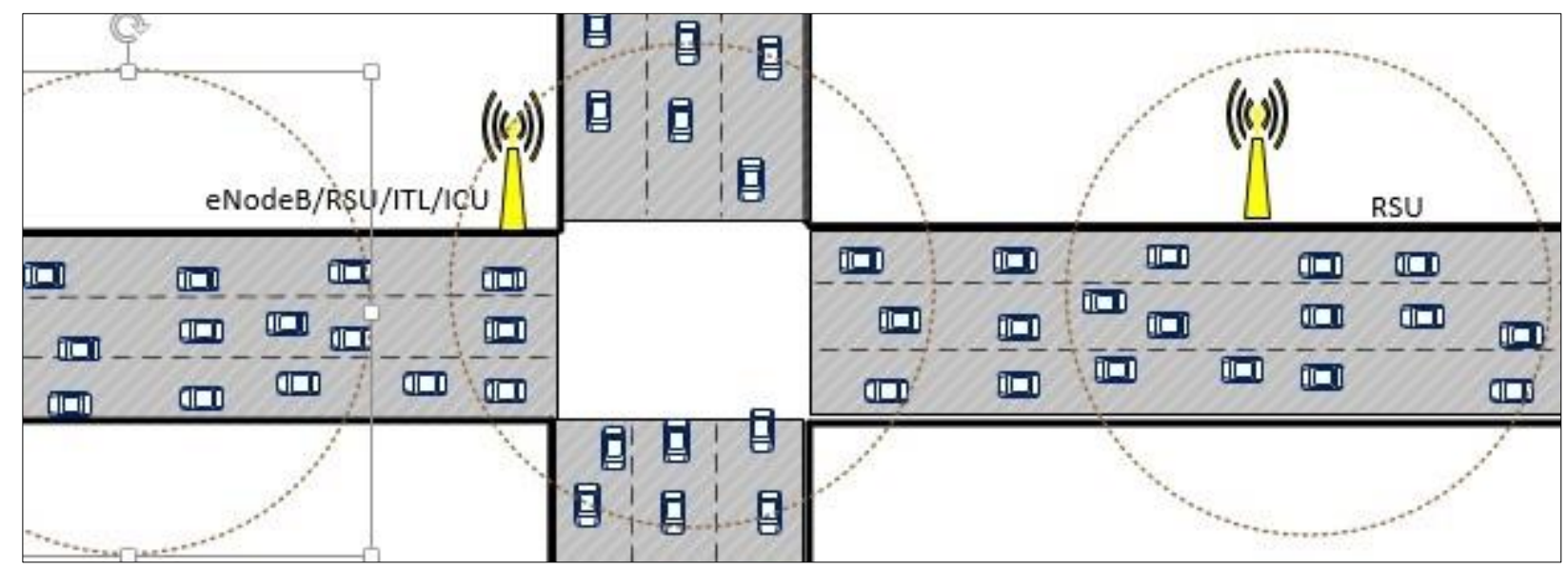

Figure 6. Infrastructure-based emergency messages dissemination(46)

The accident and congestion prevention scheme (51) is based on two components. The first component consists of the accidents avoidance algorithm and the second is congestion prevention algorithm. Affected vehicle sends emergency message to the nearest RSU. To avoid duplication, RSU will first check whether the message is previously received or not. After confirmation, it will send the message to other RSU in its proximity. In the congestion prevention process, after receiving the message from the RSU, moving vehicle will check its direction and destination and change its route based on the information sent in the emergency message. The performance of the proposed schemes is measured through delay, high delivery ratio and throughput. Although the author has tried to reduce the frequency of messages over the network, due to the complexity of the prioritization process, performance of the delivery of the messages will suffer which is not affordable for emergency based applications.

An emergency prediction and prevention system is proposed by (52) which is based on V2I and I2V communication. Prediction is performed in first three phases and prevention is done in the fourth phase. Every vehicle sends its status in term of distance, speed and yaw rate. RSU compares this status with normal values. Moreover, RSU gets the status of the traffic flow through sensed data using sensor which indicates the presence of the vehicle. After processing data from vehicles and sensor, RSU decides about emergency status or prediction about accident. In case of abnormal values, RSU sends warning messages to vehicles in its range and its neighbouring RSUs. The direction of the vehicles is not considered while calculating the road 
traffic. The system counts the vehicles which are going into the opposite direction, due to which reliability of the system is compromised.

The message dissemination scheme (53) revolves around the usage of RSU. RSU processes the information obtained through vehicles on the road. In the next step, RSU generates emergency messages based on the abnormal values of traffic. Vehicular Backbone Network (VBN) approach is used to reduce the effect of broadcast storm. RSU disseminates the emergency messages through the use of VBN. Various zones such as high, average and low are also defined. The vehicles that are directly affected by accident are in high risk zone. Proposed scheme is enhanced by increasing the notifications and reducing end-to-end delay at the cost of increased communication overhead. The performance of the proposed will suffer due to delay in forming the VBN.

An Intelligent Control Unit (ICU) based scheme is proposed by (54), considering the intersection scenario on the highway to avoid the collisions. ICU is deployed at every intersection of the highway. It continuously monitors the vehicles in its proximity. It collects vehicles information which includes location, speed and direction. ICU calculates the collision probability based on the received data and compares it with the threshold value. ICU broadcasts the messages to all vehicles based on compared threshold values. Although this scheme is most effective for highway environment, but in urban scenario, it will be a costly solution due to high number of ICU required for large number of intersections.

A congestion detection scheme (55) considers the use of driver's smart phone. Smart phone is equipped with an application that keeps the record of location using Geographic Position based System (GPS). Location information of each vehicle is sent to the remote server. Server detects congestion based on the location sent by different vehicles. Server processes the information using defined threshold values and declares the congestion in case of abnormal values. The same information is sent to all vehicles through RSU. Although this scheme has used the latest technology to detect the congestion on the road, but, when a large number of vehicles connect to the server, there are processing and information handover issues which affect the performance of the proposed system. Similarly, smart phone application did not consider the road status such as intersection while calculating the traffic density.

A Hybrid V2X scheme proposed by (56) depends on cellular network and Wi-Fi direct. It uses Wi-Fi direct for local communication links instead of WAVE/DSRC. Vehicles use cellular network for continuous connectivity. There are number of issues that should be considered before the practical implementation of the proposed system. Some of issues include the network load on the cellular networks, delay in link establishment of local links, reduction of provision time and communication of Wi-Fi direct with individual adhoc networks.

An Infrastructure based scheme (57) consists of three phases. In the first phase, all vehicles in the vicinity of RSU register themselves with their respective RSU. RSU keeps records of all vehicles in its range. In the second phase, RSU and vehicles register to Trusted Authority (TA) to create liability with TA. In the third phase which is also called execution phase, RSU broadcasts messages to communicate with the vehicles in its range. Vehicles respond with Basic Safety Message (BSM) to RSU periodically. If the distance between two vehicles moving on the same lane is less than defined safe distance, vehicles send warning messages to its following vehicle. RSU also monitors threshold values based on velocity and distance. The Message Delivery Ratio, Message Loss Ratio, Average Delay, and Basic Safety Message metrics are used to measure the performance of the proposed scheme. Generation of the warning messages depends on the distance between vehicles. There is a possibility of the fake messages generation due to sudden and frequent changes in the position of the vehicles.

Accident management system (48) uses cellular technology to disseminate the information. The proposed scheme is based on road awareness by considering roads intersection, lanes and traffic of both directions. The system is divided into five components: vehicular, central server, RSUs, ambulance and hospital. The vehicular components include sensor, message and alert, and speed monitoring module. Sensor module that controls all sensors is embedded to vehicles. It includes two types of sensors: biomedical sensors and vehicle sensors. Speed module keeps the track of vehicle's current speed and average speed. Message and alert module describes the communication between central servers, RSU and ambulance. Delay increases due to dependency on different components. As a result, system performance is affected in dense traffic.

Sensor enabled message dissemination scheme (58) exploits cloud services to provide secure message dissemination. Every vehicle shares its information such as position, speed and direction to nearest gateway. Moreover, every vehicle is registered based on unique ID and password. The 
security of the information stored in the cloud is achieved through implementing blowfish technique. The emergency messages are broadcasted to all vehicles through the cloud connection. The broadcast is performed through selecting gateways. In the proposed scheme, reliability is improved by considering registration and blowfish techniques. A message is first forwarded to cloud and broadcasted through gateway due to which extra delay is created. Moreover, maximum range of the emergency message is also affected.

The Infrastructure-based data dissemination makes use of flooding approach to distribute messages. It is quite simple as compared to other two approaches. Messages are sent with minimum delay to directly connected vehicles. It depends on the deployment on the maximum number of broadcasting devices to cover the lengths of the road. So, it is a costly solution in terms of the usage of RSU. This way, delay increases due to using large number of RSU. Similarly, delay incurs due to inter communication of the different types of the infrastructure devices. RSU gets the message from the vehicles and sensors deployed on the road before broadcasting on the network which increases delay. Majority infrastructure based schemes depends on broadcasting the message while not considering the channel condition which results in delay, collisions, packet losses, interference. So, high communication overhead is created. Similarly, it requires to improve the existing studies to increase reliability and scalability of the received messages. The analysis of the infrastructure based schemes is given in Table 3 .

Table 3. Comparative Analysis of Infrastructure based Emergency Messages Schemes

\begin{tabular}{|c|c|c|c|c|c|}
\hline $\begin{array}{c}\text { Year/ } \\
\text { Reference }\end{array}$ & Scenario & $\begin{array}{c}\text { Road } \\
\text { Awareness }\end{array}$ & Simulators & Performance Metrics & Weakness \\
\hline $2013[49]$ & Highway & No & $\mathrm{C}++$ & Driver's reaction time & Delay, High cost \\
\hline $2014[50]$ & Highway & No & $\mathrm{C}++$ & Delay, No of car crashes & $\begin{array}{l}\text { Sparse network } \\
\text { issue, reliability }\end{array}$ \\
\hline $2014[46]$ & Urban & Yes & NS2 & Average density & $\begin{array}{l}\text { Communication } \\
\text { overhead, High } \\
\text { cost, delay }\end{array}$ \\
\hline $2015[51]$ & Urban & No & NS2 & PDR, Delay, Throughput & Delay \\
\hline $2015[52]$ & Highway & No & $\mathrm{NS} 2$ & Prediction accuracy & Reliability \\
\hline $2015[53]$ & Highway & Yes & NS2 & Delay, overhead & $\begin{array}{l}\text { VBN forming } \\
\text { delay }\end{array}$ \\
\hline $2015[54]$ & Highway & Yes & OMNET++,SUMO & Collision probability & High cost \\
\hline $2016[55]$ & Urban & Yes & $\mathrm{C}++$ & Congestion detection & $\begin{array}{c}\text { Communication } \\
\text { overhead, high } \\
\text { cost }\end{array}$ \\
\hline $2016[56]$ & Highway & NO & N/M & Elapsed time & $\begin{array}{l}\text { Network load, } \\
\text { delay, interfacing } \\
\text { establishment }\end{array}$ \\
\hline $2018[57]$ & Highway & No & NS2 & $\begin{array}{c}\text { Message delivery ratio, } \\
\text { Message loss ratio, } \\
\text { Average delay }\end{array}$ & Fake alarms \\
\hline $2018[48]$ & Urban & No & MATLAB & $\begin{array}{l}\text { Average speed, time taken } \\
\text { against density }\end{array}$ & Delay \\
\hline $2019[58]$ & Highway & No & NS2, SUMO & $\begin{array}{c}\text { Packet Delivery Ratio } \\
\text { Throughput } \\
\text { Routing Control Overhead } \\
\text { Transmission Delay } \\
\text { Key Computation and Key } \\
\text { Recovery Time } \\
\end{array}$ & $\begin{array}{l}\text { delay, limited } \\
\text { dissemination } \\
\text { range }\end{array}$ \\
\hline
\end{tabular}




\section{Challenges and Open Research Problems:}

Several studies have been presented regarding emergency messages dissemination. The main focus of these proposals is to send messages with high reliability, low delay and to cover maximum range. Emergency messages dissemination has become a highly challenging task in VANET due to some specific characteristics of VANET such as sudden and frequent change in topology, high variations in density and frequent network disconnections. In the proposed classification, existing emergency messages schemes are divided into three main categories: Infrastructure-based, cluster-based and relay node-based. All these emergency messages schemes depend on when and how to broadcast the message. In the recent studies, it is tried to provide the complete emergency solution using sensors, cloud servers and IOT. Infrastructure-based approaches depend on the deployment of the infrastructure which increases the cost and also increases delay due to the dependence on these devices. Clustering approach uses V2V communication to disseminate the message. Cluster instability is the major issue of clustering. Performance of the emergency messages dissemination schemes is affected due to frequent $\mathrm{CH}$ change, frequent leaving and joining of cluster members. Several single hop and multihop emergency messages schemes have also been proposed. One of the key components in these schemes is to select the forwarder. The selection of the reliable forwarder in a dense, sparse and highly dynamic scenario is a big issue. Although relay node approach also has limitations but as compared to clustering based approach, it offers more advantages when it is used with infrastructure approach. Infrastructure needs some mechanism to disseminate the messages to large distance. In the clustering approach, in order to manage the cluster, $\mathrm{CH}$ also sends/ receives messages to/from its cluster members. Whereas in relay node, the only main task is to elect the suitable forwarder to disseminate the messages to large distance. While using relay node, scalability increases in the context of the dissemination of messages.

Using relay node approach in combination with the infrastructure improves the performance of emergency messages by decreasing cost, increasing reliability with maximum distance covered.

Moreover, Relay node based, cluster based and infrastructure based emergency messages schemes depend on underlying VANET technology. There are a number of factors that should be addressed during the design of emergency messages schemes for improving the efficiency of ITS. For future research work, this research can be improved and extended in several ways that are highlighted as follows.

\section{- VANET's Network Instability}

The performance of the ITS depends on the efficiency of emergency message dissemination techniques. This efficiency is affected due to VANET's characteristics such as frequent topological changes, high dynamicity, unexpected density variation and less connection time between vehicles $(59,60)$. Several techniques have been proposed to cater above issues but there is still need to improve the existing techniques to reduce the network instability.

\section{- Intelligent Message Dissemination}

Existing studies have tried to enhance the performance of the emergency messages schemes based on different scenarios. There is need to introduce more intelligent techniques (61) that may adapt to change in the dynamic environments of the VANET including different ratio of the traffic at different times. Moreover, there is need to calculate the density based on classifying the traffic in terms of cars, buses and bikes because one heavy truck on roads affects the flow of the traffic and creates emergency situation.

\section{- Message Security}

Emergency messages dissemination is very crucial and important information that is distributed over the whole network. Intruders disrupts the VANET communication by modifying contents, replaying sources by changing routes and destinations which increase delay between source and destination. Fake information from fake sources will also affect the reliability of the disseminated messages $(62,63)$. There is need to add the security measures while keeping the reliability, authenticity and accuracy issues in consideration.

\section{- Road Aware Emergency Messages \\ Generation}

Existing density based emergency messages schemes generate the emergency situation by estimating the number of vehicles in a specific area. These schemes depend on the threshold value of the density of the vehicles $(64,65)$. Threshold values are not formulated while considering actual status of the roads in terms of road length, width and number of lanes. Moreover, there exist situations when threshold value alarms the emergency situation in spite of sufficient resources for the smooth flow of the traffic. There is need to design emergency messages schemes based on the road status while considering density-resources ratio. 


\section{- Broadcast Storm}

A lot of efforts have already been done on controlling the broadcast, but it still needs attention while including the latest technologies and considering different scenario such as dense and sparse networks to optimize the emergency messages dissemination (66). There is need to cater the challenges such as network segmentation, overlapping areas, selection of broadcasting nodes and optimum deployment of infrastructure that affect the process of broadcasting.

\section{- Cost Effective Solutions}

Existing studies are designed in keeping the scenarios of highways and urban areas in consideration. These schemes are based on infrastructure such as RSU, ICU, ITL and sensors. These schemes exhibit excellent functionality at a reasonable cost in highway environment but they are a costly solution when implemented in urban areas due to large number of intersections $(46,47$, 52). There is need to design schemes which give cost effective solution while optimizing the performance for emergency message schemes.

\section{- Integration of Cellular Networks}

Existing studies $(56,67,68)$ integrated the cellular networks such as Long Term Evolution (LTE, 5G to connect vehicles directly with the infrastructure and Wi-Fi direct for local communications between vehicles to get real time responses. Apart from its advantages in terms of real time communication, there are still a number of open challenges that need to be considered such as the high cost of 5G communications, interfacing, handover issues and stability of Wi-Fi direct for local communication.

\section{Conclusion:}

The ITS ultimately improves the existing systems by integrating information communication technologies using VANET. This paper provides an analysis of the existing studies on emergency messages dissemination. Different studies have been proposed to highlight challenges such as broadcast problem and inefficient and unreliable delivery of the messages. These studies classify the existing schemes based on techniques such as pull, push, opportunistic, multicasting, geocasting, QOS and number of technologies which include Internet of things, software defined network, cellular networks and fog Computing. Methodology proposed in each study has its pros and cons and improves performance under different scenarios and conditions. Existing studies depend on infrastructure such as RSU, ITL, ICU and cellular networks to provide the efficient delivery of messages. Different techniques such as clustering and relay node are used to improve the performance of the message dissemination. This paper classifies the existing emergency messages schemes into main three categories: relay node, clustering and infrastructure. Moreover, it highlights advantages and disadvantages of each type based on $\mathrm{V} 2 \mathrm{~V}$ and V2I communication. In the proposed methodology, it is concluded that use of relay node based approach in combination with the infrastructure improves the performance of emergency messages by decreasing cost, increasing reliability and disseminating the messages to maximum range of the network. It will enhance the performance of the transport systems by reducing congestion and accidents, and increasing driver awareness by implementing state of the art technologies. A complete solution based on emergency prevention and information dissemination considering real time road scenarios using latest technologies may be explored in the future studies.

\section{Authors' declaration:}

- Conflicts of Interest: None.

- We hereby confirm that all the Figures and Tables in the manuscript are mine ours. Besides, the Figures and images, which are not mine ours, have been given the permission for republication attached with the manuscript.

- The author has signed an animal welfare statement.

- Ethical Clearance: The project was approved by the local ethical committee in Carleton University.

\section{References:}

1. Tewolde GS. Sensor and network technology for intelligent transportation systems. In2012 IEEE International Conference on Electro/Information Technology 2012 May 6 (pp. 1-7). IEEE..

2. Khekare GS, Sakhare AV. Intelligent traffic system for VANET: A survey. Int J Adv Compu Res. 2012;2(4):99.

3. Qureshi KN, Abdullah AH. A survey on intelligent transportation systems. Middle East J Sci Res. 2013;15(5):629-42.

4. Abbas G, Abbas ZH, Haider S, Baker T, Boudjit S, Muhammad F. PDMAC: A Priority-Based Enhanced TDMA Protocol for Warning Message Dissemination in VANETs. Sensors. 2020;20(1):45.

5. Cuyu C, Yong X, Meilin S, Liang L. Performance observations on mac protocols of vanets in intelligent transportation system. 2009 WRI International Conference on Communications and Mobile Computing; 2009: IEEE.

6. Al Najada H, Mahgoub I. Anticipation and alert system of congestion and accidents in VANET using Big Data analysis for Intelligent Transportation 
Systems. 2016 IEEE Symposium Series on Computational Intelligence (SSCI); 2016: IEEE.

7. Schoch E, Kargl F, Weber M, Leinmuller $T$. Communication patterns in VANETs. IEEE Commun Mag. 2008;46(11):119-25.

8. Darwish T, Bakar KA. Traffic aware routing in vehicular ad hoc networks: characteristics and challenges. Telecommun Syst. 2016;61(3):489-513.

9. Jindal V, Bedi P. Vehicular ad-hoc networks: introduction, standards, routing protocols and challenges. IJCSI. 2016;13(2):44.

10. Perallos A, Hernandez-Jayo U, Zuazola IJG, Onieva E. Intell Transp Syst Tech Appl. John Wiley \& Sons; 2015.

11. Younes MB, Boukerche A. A performance evaluation of an efficient traffic congestion detection protocol (ECODE) for intelligent transportation systems. Ad Hoc Netw. 2015;24:317-36.

12. Huo Y, Liu Y, Ma L, Cheng X, Jing T. An enhanced low overhead and stable clustering scheme for crossroads in VANETs. EURASIP J Wirel Comm. 2016(1):74.

13. Zhang D, Ge H, Zhang T, Cui Y-Y, Liu X, Mao G. New multi-hop clustering algorithm for vehicular ad hoc networks. IEEE Trans Intell Transp Syst. 2018;20(4):1517-30.

14. Ahmad I, Noor RM, Ahmedy I, Shah SAA, Yaqoob I, Ahmed E, et al. VANET-LTE based heterogeneous vehicular clustering for driving assistance and route planning applications. Comput Netw. 2018;145:128-40.

15. Mammu ASK, Hernandez-Jayo U, Sainz N. Clusterbased MAC in VANETs for safety applications. 2013 International Conference on Advances in Computing, Communications and Informatics (ICACCI); 2013: IEEE.

16. Yang F, Tang Y, Huang L. A multi-channel cooperative clustering-based MAC protocol for VANETs. 2014 Wirel Telecommun Symp; 2014: IEEE.

17. Sakiz F, Sen S. A survey of attacks and detection mechanisms on intelligent transportation systems: VANETs and IoV. Ad Hoc Netw. 2017;61:33-50.

18. Kumar R, Dave M. A review of various vanet data dissemination protocols. IJUNESST. 2012;5(3):2744.

19. Chaqfeh M, Lakas A, Jawhar I. A survey on data dissemination in vehicular ad hoc networks. Veh Commun. 2014;1(4):214-25.

20. Kaur N, Singh A. A survey on data dissemination protocols used in VANETs. Int $\mathrm{J}$ Comput Appl. 2015;120(23).

21. Sanguesa JA, Fogue M, Garrido P, Martinez FJ, Cano J-C, Calafate CT. A survey and comparative study of broadcast warning message dissemination schemes for VANETs. Mob Inf Syst. 2016;2016.

22. Ghebleh R. A comparative classification of information dissemination approaches in vehicular ad hoc networks from distinctive viewpoints: A survey. Comput Netw. 2018;131:15-37.

23. Ghazi MU, Khattak MAK, Shabir B, Malik AW, Ramzan MS. Emergency Message Dissemination in
Vehicular Networks: A Review. IEEE Access. 2020;8:38606-21.

24. Eze EC, Zhang S, Liu E. Vehicular ad hoc networks (VANETs): Current state, challenges, potentials and way forward. 2014 20th International Conference on Automation and Computing; 2014: IEEE.

25. Chitra M, Sathya SS. Efficient broadcasting mechanisms for data dissemination in vehicular ad hoc networks. IJMNCT. 2013;3(3):47-63.

26. Gawas MA, Hurkat P, Goyal V, Gudino LJ. Cross layer approach for efficient dissemination of emergency messages in VANETs. 2017 Ninth International Conference on Ubiquitous and Future Networks (ICUFN); 2017: IEEE.

27. Wang W, Luo T, Kang H. A Local Information Sensing-Based Broadcast Scheme for Disseminating Emergency Safety Messages in IoV. Mob Inf Syst. 2019;2019.

28. Dawood HS, Wang Y. An efficient emergency message broadcasting scheme in vehicular ad hoc networks. Int J Distrib Sens N. 2013;9(11):232916.

29. Huang J, Huang Y, Wang J. Vehicle density based forwarding protocol for safety message broadcast in VANET. Sci World J. 2014;2014.

30. Fogue M, Martinez FJ, Garrido $P$, Fiore $M$, Chiasserini C-F, Casetti C, et al. Securing warning message dissemination in VANETs using cooperative neighbor position verification. IEEE Trans Veh Technol. 2014;64(6):2538-50.

31. Rayeni MS, Hafid A, Sahu PK. Dynamic spatial partition density-based emergency message dissemination in VANETs. Veh Commun. 2015;2(4):208-22.

32. Zemouri S, Djahel S, Murphy J. A fast, reliable and lightweight distributed dissemination protocol for safety messages in Urban Vehicular Networks. Ad Hoc Netw. 2015;27:26-43.

33. Shaik S, Ratnam DV, Bhandari B. An efficient cross layer routing protocol for safety message dissemination in VANETS with reduced routing cost and delay using IEEE 802.11 p. Wirless Pers Commun. 2018;100(4):1765-74.

34. Selvi M, Ramakrishnan B. An Efficient Message Prioritization and Scheduled Partitioning Technique for Emergency Message Broadcasting in VANET. 2018 3rd International Conference on Communication and Electronics Systems (ICCES); 2018: IEEE.

35. Benrhaiem W, Hafid A, Sahu PK. Reliable Emergency Message Dissemination Scheme for Urban Vehicular Networks. IEEE Trans Intell Transp Syst. 2019.

36. Mchergui A, Moulahi T, Othman MTB, Nasri S. Enhancing VANETs Broadcasting Performance with Mobility Prediction for Smart Road. Wirless Pers Commun. 2020:1-13.

37. Ghodrati AD. Reduces broadcast storm using clustering in VANETs. IRJABS. 2013;7(13):979-87.

38. Benkerdagh S, Duvallet C. Cluster - based emergency message dissemination strategy for VANET using V2V communication. Int J Commun Syst . 2019;32(5):e3897. 
39. Jin D, Shi F, Song J. Cluster based emergency message dissemination scheme for vehicular ad hoc networks. Proceedings of the 9th International Conference on Ubiquitous Information Management and Communication; 2015.

40. Ramakrishnan B, Nishanth RB, Joe MM, Selvi M. Cluster based emergency message broadcasting technique for vehicular ad hoc network. Wirel Netw. 2017;23(1):233-48.

41. Cambruzzi E, Farines J-M, Kraus W, Macêdo R. A Cluster Management System for VANETs. Int J Intell Transp Syst Res. 2016;14(2):115-26.

42. Ramakrishnan B, Selvi M, Nishanth RB, Joe MM. An emergency message broadcasting technique using transmission power based clustering algorithm for vehicular ad hoc network. Wirless Pers Commun. 2017;94(4):3197-216.

43. Shah SS, Malik AW, Rahman AU, Iqbal S, Khan SU. Time Barrier-Based Emergency Message Dissemination in Vehicular Ad-hoc Networks. IEEE Access. 2019;7:16494-503.

44. Ali M, Malik AW, Rahman AU, Iqbal S, Hamayun MM. Position-based emergency message dissemination for Internet of vehicles. Int J Distrib Sens N. 2019;15(7):1550147719861585.

45. Patil SD, Ragha L. Adaptive fuzzy-based message dissemination and micro-artificial bee colony algorithm optimised routing scheme for vehicular ad hoc network. IET Commun. 2020;14(6):994-1004.

46. Khekare GS. Design of emergency system for intelligent traffic system using VANET. International Conference on Information Communication and Embedded Systems (ICICES2014); 2014: IEEE.

47. Raut SB, Bajaj PR, Malik LG. Prediction of vehicle collision probablity at intersection using V2V communication. Int J Sci Eng Res. 2015;6(5):295300.

48. Al-Mayouf YRB, Mahdi OA, Taha NA, Abdullah NF, Khan S, Alam M. Accident Management System Based on Vehicular Network for an Intelligent Transportation System in Urban Environments. J Adv Transport; 2018.

49. Garcia-Lozano E, Barba CT, Igartua MA, Campo C. A distributed, bandwidth-efficient accident prevention system for interurban VANETs. 2013 International Conference on Smart Communications in Network Technologies (SaCoNeT); 2013: IEEE.

50. Bharath A, Sivagurunathan P. Collision Avoidance system in vehicular adhoc network utilizing dichotomized headway model. 2014 International Conference on Circuits, Power and Computing Technologies (ICCPCT-2014); 2014: IEEE.

51. Roy A, Chakraborty J. Communication based accident avoidance and congestion control mechanism in VANETs. 2015 International Symposium on Advanced Computing and Communication (ISACC); 2015: IEEE.

52. Ganeshkumar P, Gokulakrishnan P. Emergency situation prediction mechanism: a novel approach for intelligent transportation system using vehicular ad hoc networks. The scientific world journal. 2015;2015.

53. Gokulakrishnan P, Ganeshkumar P. Road accident prevention with instant emergency warning message dissemination in vehicular ad-hoc network. PloS one. 2015;10(12):e0143383.

54. Raut SB, Bajaj PR, Malik LG. Prediction of vehicle collision probablity at intersection using V2V communication. Int J Sci Eng Res. 2015;6:295-300.

55. Jayapal C, Roy SS. Road traffic congestion management using VANET. 2016 International Conference on Advances in Human Machine Interaction (HMI); 2016: IEEE.

56. Jeong S, Baek Y, Son SH. A hybrid V2X system for safety-critical applications in VANET. 2016 IEEE 4th International Conference on Cyber-Physical Systems, Networks, and Applications (CPSNA); 2016: IEEE.

57. Aldegheishem A, Yasmeen H, Maryam H, Shah M, Mehmood A, Alrajeh N, et al. Smart road traffic accidents reduction strategy based on intelligent transportation systems (tars). Sensors. 2018;18(7):1983.

58. Sathya NP. A sensor enabled secure vehicular communication for emergency message dissemination using cloud services. Digit Signal Process. 2019;85:10-6.

59. Abboud K, Zhuang W. Impact of microscopic vehicle mobility on cluster-based routing overhead in VANETs. IEEE Trans Veh Technol. 2015;64(12):5493-502.

60. Abuashour A, Kadoch M. Performance improvement of cluster-based routing protocol in VANET. IEEE Access. 2017; 5:15354-71.

61. Cunha F, Villas L, Boukerche A, Maia G, Viana A, Mini RA, et al. Data communication in VANETs: Protocols, applications and challenges. Ad Hoc Netw. 2016; 44:90-103.

62. Sun C, Liu J, Xu X, Ma J. A privacy-preserving mutual authentication resisting DoS attacks in VANETs. IEEE Access. 2017;5: 24012-22.

63. Lu Z, Qu G, Liu Z. A survey on recent advances in vehicular network security, trust, and privacy. IEEE Trans Intell Transp Syst. 2018; 20(2): 760-76.

64. Eze EC, Zhang S-J, Liu E-J, Eze JC. Advances in vehicular ad-hoc networks (VANETs): Challenges and road-map for future development. IJAC. 2016;13(1): 1-18.

65. Mehmood A, Khanan A, Mohamed AHH, Mahfooz S, Song H, Abdullah S. ANTSC: An intelligent Naïve Bayesian probabilistic estimation practice for traffic flow to form stable clustering in VANET. IEEE Access. 2017; 6:4452-61.

66. Feukeu E, Zuva T. Dynamic broadcast storm mitigation approach for VANETs. Future Gener Comp Sy. 2018.

67. Lai C, Zheng D, Zhao Q, Jiang X. SEGM: A secure group management framework in integrated VANET-cellular networks. Veh Commun. 2018;11:33-45.

68. Wang P, Di B, Zhang H, Bian K, Song L. Cellular V2X communications in unlicensed spectrum: 
Harmonious coexistence with VANET in 5G

systems. IEEE Trans Wireless Commun.

2018;17(8):5212-24.

\section{تحديات نشر رسائل الطوارئ من خلال المركبات المتصلة لأنظمة النقل الذكية الفعالة: مراجعة}

فاطمة طول الزهرة

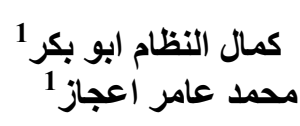

1قسم علوم الحاسبات، كلية الهندسة الجامعة التكنولوجية الماليزية، ماليزيا

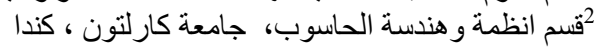

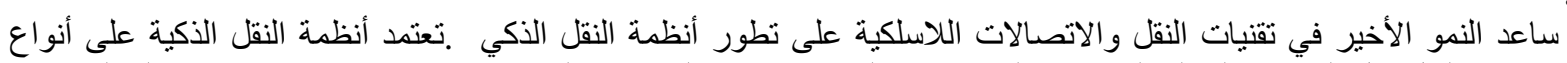

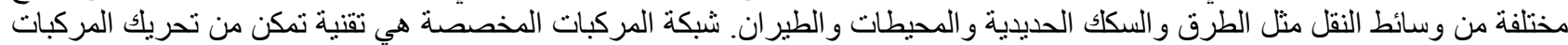

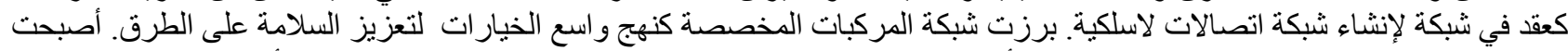

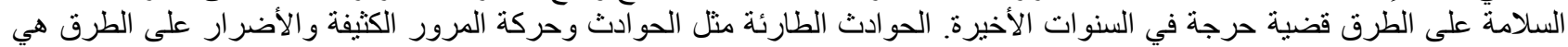

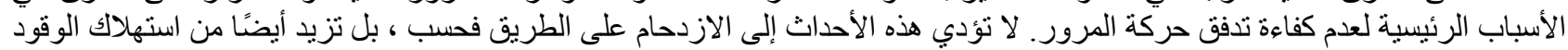

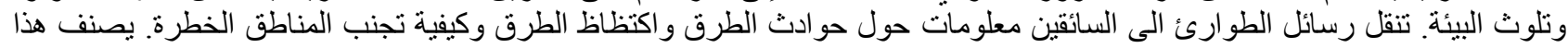

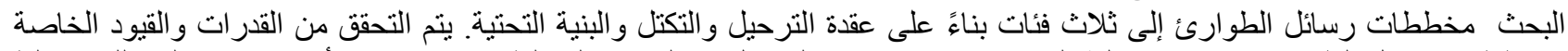

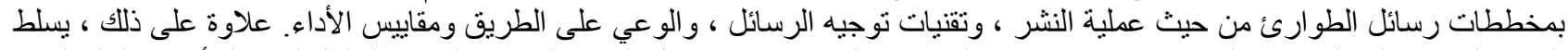

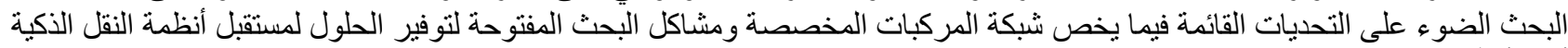
أكثر أمانًا وفعالية واستدامة.

الكلمات المفتاحية : الحو ادث، السلامة على الطرق، أنظمة النقل الذكية (ITS)، رسائل الطو ارئ، شبكة المركبات المخصصة( VANET ) 